E3S Web of Conferences 2, 03006 (2014)

DOI: $10.1051 /$ e3sconf/ 20140203006

(C) Owned by the authors, published by EDP Sciences, 2014

\title{
Aree Protette del Po e della Collina Torinese: studi propedeutici alla Candidatura MAB - Man and the Biosphere ${ }^{1}$
}

\section{Nature Reserves of the Po river and of the Turin hills: preliminary studies for the candidacy to MAB - Man and the Biosphere}

\author{
Elisabetta Cimnaghi ${ }^{1}$, Annamaria Delmonte ${ }^{1}$, Elisa Zanetta ${ }^{1}$, Ippolito Ostellino ${ }^{2}$ \\ ${ }^{1}$ SiTI, Istituto Superiore sui Sistemi Territoriali per l'Innovazione, via Pier Carlo Boggio 61, Torino \\ ${ }^{2}$ Ente Aree Protette del Po e della Collina Torinese, Corso Trieste, 98 - Moncalieri (TO)
}

\begin{abstract}
Riassunto. Il programma MAB fu lanciato dall'UNESCO negli anni '70 al fine di migliorare il rapporto tra uomo e ambiente. Negli anni il programma ha portato al riconoscimento di Riserve della Biosfera che gli Stati Membri si impegnano a gestire nella prospettiva della conservazione delle risorse. L'Organismo Gestore dell'Area Protetta, partendo, da un lato, dalla consapevolezza della ricchezza ambientale che caratterizza il suo territorio e, dall'altro, del contesto di sviluppo antropico elevato in cui essa è situata, ha riconosciuto nel programma $\mathrm{MAB}$ un'opportunità per incrementare le sue attività di protezione e potenziamento delle risorse. Questo articolo descrive i primi risultati ottenuti negli studi preliminari per la Designazione al MAB, con particolare attenzione sia alle opportunità di implementazione di progetti legati al marchio "Collina Po", creato dall'Organo di Gestione del Parco, si ain termini di scambio di 'best practices' con altre Riserve di Biosfera.
\end{abstract}

\begin{abstract}
The MAB programme was established by UNESCO in the $70 \mathrm{~s}$ in order to improve the relationship between man and environment. Over the years, the programme has led to the recognition of Biosphere Reserves that Member States are committed to manage in the perspective of resources conservation. The Management Body of the Protected Area, starting from the awareness of environmental richness characterizing its territory on one hand and of the context of high anthropic development in which it is
\end{abstract}

Il contributo è frutto del lavoro congiunto dei quattro autori. Pur essendo la responsabilità scientifica attribuibile a tutti e quattro, l'abstract e il paragrafo 1 sono da attribuire al dott. Ostellino, i paragrafi 2 e 6 sono stati curati ng. Elisabetta Cimnaghi, il paragrafo 3 dalla dott.ssa Annamaria Delmonte e il paragrafo 4 dall'arch. Elisa Zanetta. Il paragrafo 5 è da attribuire congiuntamente alla dott.ssa Delmonte e all'arch. Zanetta. dall'i 
located on the other hand, has recognized in the MAB Programme a chance to increase its activities of protection and enhancement of the resources. This paper reports the first results achieved in the preparatory studies for the Nomination to MAB, with special attention to the opportunities both of implementation of projects related with the brand "Collina Po" created by the Park Management Body and in terms of exchange of best practices with other Reserves of Biosphere.

\section{Introduzione}

Il Programma MAB è stato avviato dall'UNESCO negli anni ' 70 allo scopo di migliorare il rapporto tra uomo e ambiente. Negli anni, esso ha portato al riconoscimento delle Riserve della Biosfera che gli Stati membri s'impegnano a gestire nell'ottica della conservazione delle risorse.

In questo contesto si inserisce una proposta progettuale dell'Ente Aree Protette del Po e della Collina Torinese che, sulla base della consapevolezza della ricchezza ambientale caratterizzante il proprio territorio da un lato e del contesto ad alto sviluppo antropico nel quale esso è collocato dall'altro, ha visto nel Programma $\mathrm{MAB}$ una possibilità di incrementare le proprie attività di tutela e di valorizzazione delle risorse.

Scopo del presente lavoro è rendere conto dei primi risultati ottenuti nell'ambito di studi propedeutici alla Candidatura MAB per il Parco, con un'attenzione particolare alle opportunità per l'attuazione delle progettualità individuate negli ultimi anni dall'ente per il territorio definito anche dal proprio brand Collina Po e in termini di scambio di best practices che ne potrebbero derivare.

\section{II ruolo delle aree protette nello sviluppo di un territorio}

Le problematiche relative allo sviluppo sostenibile richiedono una profonda revisione del modello di sviluppo finora adottato e mettono in campo la complessità del tema del governo del territorio, inteso come attività di programmazione e gestione integrata delle risorse naturali, del patrimonio culturale, del paesaggio, dell'assetto insediativo, dello sviluppo locale. In questo contesto si inseriscono le aree protette (AP), quali soggetti attivi nel processo di governo del territorio: l'obiettivo "storico" delle AP, quello di conservazione della biodiversità, sembra non essere più sufficiente e piuttosto si richiede loro un importante contributo per lo sviluppo sociale ed economico dei luoghi e delle popolazioni ad esse correlati.

In particolare, il percorso per giungere ad una definizione univoca di "area protetta" è stato lungo e complesso, al punto che tutt'ora non si può considerare concluso [1-11].

Recentemente, l'International Union for Conservation of Nature (IUCN) ha definito, ottenendo generali consensi nel mondo scientifico, le aree protette come «a clearly defined geographical space, recognized, dedicated and managed to achieve the long-term conservation of nature, associated ecosystem services and cultural values» ${ }^{2}$. Si tratta di una definizione ricca di significati, in quanto racchiude in sé i risultati del lungo processo evolutivo che ha visto modificare nel tempo il ruolo delle AP nella tutela delle risorse naturali e nel governo del territorio. Da luoghi esclusivamente dedicati alla protezione dell'ambiente, i cosiddetti "santuari della natura" per usare un termine ormai obsoleto, i parchi e le riserve sono oggi territori improntati allo sviluppo economico e culturale.

Secondo questa nuova interpretazione, le politiche di gestione delle AP devono sempre più indirizzarsi alla diffusione dei benefici della protezione al di là dei loro confini amministrativi, promuovendosi come centri di programmazione e motori dello sviluppo sostenibile nei contesti allargati di

\footnotetext{
2 [8, pag. 8].
} 
appartenenza, attraverso la condivisione delle politiche con le popolazioni insediate. Ne emerge un quadro in cui le AP sono punto di riferimento per progetti di valorizzazione culturale, ambientale, economica e sociale, secondo una visione nella quale è richiesto un graduale superamento delle politiche di protezione sviluppate per ogni singola area protetta a favore della costruzione di un sistema integrato. In particolare, il fine ultimo di tale processo deve essere la costruzione di reti capaci di collaborare tra di loro.

Affinché il modello di gestione delle AP sia effettivamente in grado di fornire un contributo positivo allo sviluppo di un territorio, è necessario un costante confronto con le dinamiche che lo riguardano, quali ad esempio la crescita del turismo, gli effetti del cambiamento climatico, il crescente fenomeno di abbandono delle terre in aree montane.

Se, come è stato affermato, il ruolo delle aree protette non è solo più quello di conservare, ma piuttosto quello di rendere fruibile un territorio, diventa necessario prevedere strumenti di gestione che siano in grado di permettere ciò senza compromettere il valore delle risorse presenti: sono quindi sempre più fondamentali gli sforzi finalizzati a definire adeguatamente la mission delle aree protette, attraverso un costante e fondamentale dialogo tra gli stakeholder che sappia garantire equilibrio e collaborazione tra le parti coinvolte.

\section{II programma MAB, Man and the Biosphere}

Il Programma MAB - Man and the Biosphere - è stato avviato dall'UNESCO negli anni '70 allo scopo di migliorare il rapporto tra uomo e ambiente e ridurre la perdita di biodiversità attraverso programmi di ricerca e capacity-building. Esso ha portato al riconoscimento delle Riserve della Biosfera con la finalità di promuovere e sostenere un rapporto equilibrato fra la comunità umana e gli ecosistemi e creare siti privilegiati per la ricerca, la formazione e l'educazione ambientale, oltre che poli di sperimentazione di politiche mirate di sviluppo e pianificazione territoriale.

Le Riserve di Biosfera sono aree abitate e sfruttate dall'uomo, rimaste in uno stato vicino a quello naturale grazie alla loro economia agraria o forestale; in ragione di questa loro peculiarità, esse puntano non solo ad incoraggiare la conservazione degli spazi naturali ma anche a promuovere il territorio, il suo sviluppo economico e le sue specificità culturali.

L'obiettivo di conciliazione tra istanze di sviluppo antropico e tutela della biodiversità degli ecosistemi viene perseguito attraverso l'individuazione di tre zone: una zona centrale in cui viene preservata la biodiversità vegetale e animale, destinata alla ricerca (Core Area); una zona cuscinetto di gestione ecologica per le attività a basso impatto in termini di silvicoltura, agricoltura ecologica ed ecoturismo (Buffer Zone); una zona di sviluppo sostenibile delle risorse per l'artigianato, i servizi e le attività agro-silvo-pastorali più estensive (Transition Area).

Nel suo complesso ogni Riserva ha lo scopo di soddisfare tre funzioni complementari:

- una funzione di conservazione volta alla conservazione dei paesaggi, degli habitat, degli ecosistemi, così come delle specie e della diversità genetica;

- una funzione di sviluppo, per favorire lo sviluppo economico e umano e generare non solo reddito, ma sostenibilità socio-culturale ed ambientale nel lungo periodo;

- una funzione logistica e di supporto al fine di promuovere la comprensione dello sviluppo sostenibile e assicurare sostegno alla ricerca, monitoraggio e formazione a livello locale, oltre i confini della riserva della biosfera e attraverso lo scambio globale di buone pratiche.

In questo senso le Riserve della biosfera possono essere considerate come siti di eccellenza in cui pratiche innovative per la gestione della natura e delle attività umane sono testate e dimostrate; inoltre, sono luoghi in ci si cerca di conciliare la conservazione della diversità biologica e culturale e lo sviluppo socio-economico attraverso partnership tra le persone e la natura. Infine, sono da considerarsi strumenti di supporto per mettere in atto le indicazioni derivanti dalla Convenzione sulla diversità biologica in termini di approccio ecosistemico.

L'adozione della cosiddetta "Strategia di Siviglia" [12] ha segnato un momento decisivo per il rilancio delle Riserve di Biosfera, focalizzando l'attenzione sull'implementazione della Rete Globale delle 
Riserve di Biosfera (WNBR - World Network of Biosphere Reserves), creata nel 1976, il cui ruolo era stato fino a quel momento marginale. In risposta alle crescenti e delicate sfide ambientali del nuovo millennio, il "Madrid Action Plan" (MAP) [13] ha inoltre definito azioni, obiettivi, indicatori di risultato, partenariati ed altre modalità di attuazione del Programma MAB e della Rete Globale delle Riserve della Biosfera per il periodo 2008-2013. In questo senso le Riserve di Biosfera adottano metodi scientifici e piani di azione nazionali che si inseriscono in un quadro strategico globale volto al raggiungimento della mission del Programma.

La Rete Mondiale delle Riserve della Biosfera conta attualmente 621 riserve della biosfera in 117 paesi di tutto il mondo con una localizzazione prevalente delle iscrizioni nella regione UNESCO maggiormente sviluppata, Europa e Nord America, seguita dall'Asia e Pacifico e dall'America Latina e Caraibi. Per quanto concerne il contesto italiano, ad oggi sono registrate 8 Riserve di Biosfera: Collemeluccio-Montedimezzo (1977), Circeo (1977), Miramare (1979), Cilento e Vallo di Diano (1997), Somma-Vesuvio e Miglio d'Oro (1997), Valle del Ticino (2002), Isole della Toscana, (2003), Selva Pisana (2004). Inoltre, 6 aree stanno seguendo il processo volto ad ottenere il riconoscimento del programma MAB ed entrare nella Rete delle Riserve. Questo evidenzia, in ambito nazionale, la crescente importanza attribuita alle aree protette nella pianificazione e gestione delle attività naturali ed antropiche che trova, nella Riserva di Biosfera, un'opportunità concreta di implementazione di questa funzione. Tuttavia, questa attenzione verso le Riserve deve affiancarsi, necessariamente, al tema delle gestione: le Riserve sino ad ora riconosciute sono gestite prevalentemente in maniera diretta dagli Enti Parco che, notoriamente, stanno vivendo una fase di grande complessità particolarmente sotto l'aspetto economico. In tal senso, le nuove proposte di Riserve di Biosfera dovranno, inevitabilmente, promuovere nuove forme di gestione più svincolate rispetto agli Enti Parco, senza naturalmente prescindere dal ruolo che questi svolgono, in linea con quanto già si evidenzia nel contesto internazionale.

\section{Aree Protette del Po e della Collina Torinese: caratteristiche e peculiarità}

Il Parco del Po nasce nel 1990 a tutela dell'intero tratto fluviale piemontese e si snoda per $235 \mathrm{~km}$ dalle sue sorgenti a Pian del Re, fino alla Lombardia. A livello amministrativo la tutela del Po è affidata a tre enti, cuneese, torinese ed alessandrino - vercellese, di cui quello torinese costituisce la parte centrale. Dopo la riorganizzazione effettuata dall'entrata in vigore in Piemonte della Legge Regionale n. 19 del 29 giugno 2009 "Testo unico sulla tutela delle aree naturali e della biodiversità" oltre alla fascia fluviale nel tratto torinese sono stati riuniti in un'unica organizzazione anche i progetti di tutela relativi all'area della Collina Torinese. Oggi le Aree Protette del Po e della Collina Torinese si estendono per una superficie totale di oltre cinquemila ettari coinvolgendo tre province e trentasette comuni.

Il territorio protetto risulta complesso e diversificato: comprende in primo luogo grandi centri urbani, Torino, Moncalieri, San Mauro, Chivasso con l'infrastrutturazione e le forti pressioni antropiche che che ne derivano, ma al contempo anche paesaggi di alta qualità percettiva, luoghi ricchi di testimonianze storiche e ambienti di elevata qualità naturalistica e di biodiversità. Infatti lungo le fasce fluviali si conserva una porzione importante dei valori ecologici e della biodiversità [14].

Queste peculiarità ne fanno un caso esemplare all'interno del panorama delle aree protette italiane; proprio per la sua natura strettamente metropolitana e per i territori coinvolti fortemente permeati nei tessuti urbani e periurbani della conurbazione torinese, il parco si è posto sin dalla sua istituzione quale soggetto e strumento di salvaguardia e di valorizzazione delle risorse ambientali, naturalistiche e paesaggistiche del territorio del fiume [15-17]. Al contempo la qualità dell'ambiente naturale e dell'ambiente urbano presentano un elevato potenziale di miglioramento che negli ultimi anni ha visto l'area protetta al centro di un nuovo e forte progetto di sistema del territorio e del suo contesto 
orientato alla definizione di nuovi scenari di integrazione tra i principi di tutela e valorizzazione del patrimonio naturale e quelli di crescita economica e sociale.

\section{Proposte progettuali per il Parco in ottica MAB}

Come emerso in precedenza le Aree Protette del Po e della Collina Torinese si configurano ad oggi come entità fortemente attiva nello sviluppo di azioni rispondenti alle funzioni che il programma MAB prevede di sviluppare oltre, naturalmente, alle attività di conservazione degli ecosistemi, delle specie e della biodiversità, che fanno parte della natura stessa dell'area protetta e che, in una Riserva di Biosfera devono essere perseguite all'interno della core area. Il Parco, infatti, opera ormai da tempo, oltre che nell'ottica della salvaguardia dei valori ambientali e storico - culturali anche nella proposta di iniziative che non si fermano ai confini amministrativi dell'area protetta. Tra queste Corona Verde, finalizzata all'integrazione delle aree protette e agricole della frangia periurbana dell'area metropolitana torinese, la partecipazione ai tavoli di concertazione territoriale o le attività di costruzione di marche territoriali che contribuiscono a far crescere la visione di insieme dell'area.

Il Parco, inoltre, opera una consistente azione nel settore della formazione a livello locale e sovra locale con attività di educazione ambientale, sensibilizzazione pubblica, promozione e valorizzazione. In questo modo, fornisce supporto al fine di promuovere la comprensione dello sviluppo sostenibile, assicurando sostegno alla ricerca, al monitoraggio e alla formazione oltre i confini territoriali dell'area protetta e incentivando lo scambio di buone pratiche.

Le Aree Protette del Po e della Collina Torinese rappresentano, ad oggi, un contesto di estremo interesse per intraprendere il percorso che le qualifichi come Riserva di Biosfera. Questo non solo perché i processi sino ad oggi operati sono stati virtuosi, all'interno delle complesse congiunture politico-economiche che le aree protette stanno attraversando, ma anche perché la sua localizzazione, lungo un asse fluviale urbano, necessita di una nuova lettura nel contesto di sviluppo urbano da cui non può prescindere e con cui deve integrarsi.

In questo senso, la candidatura a Riserva di Biosfera si configura, diversamente da quanto accade per altri riconoscimenti dell'UNESCO, quale l'inserimento nella celebre Lista del Patrimonio Mondiale, non come un punto di arrivo il cui raggiungimento garantisca di per sé dei benefici quanto come un processo il cui valore risiede nella possibilità di attuare una riflessione sul proprio ruolo e sulle linee strategiche che si intendono adottare, a partire da un contesto consolidato e dalla valutazione delle criticità, in questo caso sia come area protetta sia come parco urbano. In questo senso il percorso che conduce alla proposta di Riserva intende affrontare alcune tematiche che sono, per le Aree Protette del Po e della Collina Torinese, attualmente nodali.

Tra queste spicca la gestione ambientale, non come gestione dell'ambiente in quanto tale ma, piuttosto, come gestione delle interazioni che le società umane hanno con l'ambiente; essa deve quindi includere l'economia applicata e tutti gli strumenti tecnologici, istituzionali, sociali ed empirici necessari per la tutela e il miglioramento dell'ambiente nel quadro dello sviluppo delle società umane che in tale ambiente vivono. In questo senso la gestione, attuata attraverso un piano, e regolata da un Comitato di Gestione, deve coinvolgere non solo i soggetti direttamente responsabili del patrimonio ambientale e culturale, ma anche coloro che hanno istanze diverse - soprattutto - nel caso in cui esistano conflitti fra gli stakeholder, emergenti da interessi di natura diversa.

Inoltre, una efficace tutela ambientale deriva da un flusso di risorse costante che, oggi, deve progressivamente svincolarsi dai bilanci pubblici regionali, pena l'impossibilità della stessa area di svolgere un ruolo attivo con una pianificazione a lungo termine. Nuove forme di auto-finanziamento, accanto alle risorse internazionali, devono dunque essere esplorate per contribuire al bilancio operativo di un piano di gestione, traendo suggerimento dalle esperienze maturate in altri contesti globali dalle aree naturali che operano in un quadro di sostenibilità economica. 


\section{Conclusioni}

Come si è detto, il Parco Aree Protette del Po e della Collina Torinese ha recentemente dato vita ad una serie di studi propedeutici alla Candidatura MAB per il Parco, finalizzati a comprendere se tale percorso risulti "adatto" al territorio di riferimento e se possa considerarsi utile in un'ottica di costante miglioramento delle performance gestionali dell'AP. In particolare, considerate le peculiarità del parco - parco urbano caratterizzato da risorse ad alto valore naturale e culturale - si ritiene che tale strumento possa costituire una valida opportunità in termini di sistematizzazione di quanto già fatto $\mathrm{e}$ di definizione di linee strategiche future. Se, infatti il riconoscimento MAB può essere il punto di partenza di nuove progettualità, è altrettanto vero che sono già molte e consolidate le attività svolte dal Parco che possono definirsi perfettamente aderenti alle indicazioni di tutela e valorizzazione del territorio imposte per le Riserve della Biosfera.

\section{Bibliografia}

1. E. Cimnaghi, Il ruolo delle aree protette nello sviluppo di un territorio, atti della XXXI Conferenza Italiana di Scienze Regionali, Aosta 20-22 settembre 2010 (2010).

2. R. Gambino, D. Talamo, F. Thomasset (a cura di), Parchi d'Europa, verso una politica europea per le aree protette, Edizioni ETS, Pisa (2008).

3. R. Gambino, Parchi e paesaggi d'Europa, Un programma di ricerca territoriale, Lectio Magistralis, 8 ottobre 2009, Torino (2009).

4. International Union for Conservation of Nature (IUCN), Guidelines for protected area management categories, IUCN and The World Conservation Monitoring Centre, Gland, Switzerland and Cambridge, UK, (1994).

5. IUCN, V IUCN World Parks Congress, Benefits beyond Boundaries, 8-17 September 2003, Durban, South Africa, (2003).

6. IUCN, III World Conservation Congress, People and nature, Only One World, Bangkok, 17 - 25 November 2004, Thailand, (2004).

7. IUCN -WCPA, Categories Summit, 7 - 11 May 2007, Almeria, (2007).

8. IUCN, Guidelines for applying protected area management categories, Dudley Editor, Switzerland, (2008a).

9. IUCN, IV World Conservation Congress, A diverse and sustainable world, 5-14 October 2008, Barcelona, 2008b.

10. IUCN, The IUCN Programme 2009-2012, Shaping a sustainable future, Barcellona, (2008c).

11. Phillips, Turning Ideas on their Head. The New Paradigm for Protected Areas, Durban, (2003).

12. UNESCO (United Nations Educational, Scientific and Cultural Organisation), Madrid Action Plan for Biosphere Reserves (2008-2013), (2008).

13. UNESCO (United Nations Educational, Scientific and Cultural Organisation), The Seville Strategy \& the Statutory Framework of the World Network, (1996).

14. I. Ostellino, Un parco fluviale intorno a Torino: dal Fiume Po al progetto di area metropolitana "Corona Verde", in Parchi metropolitani, A. Cavaliere e I. Ostellino, (a cura di) Edizioni ETS Pisa, (2010).

15. Ente di gestione del Parco fluviale del Po torinese. Dal bilancio sociale 2005 ai progetti per il futuro (2009).

16. I. Ostellino (a cura di), Atlante del Parco Fluviale del Po Torinese. 15 Anni di Attività fra Luoghi, Piani e Buone Pratiche, Alinea Editrice, Firenze (2005). 
Science and the Future

17. Osservatorio del Paesaggio dei Parchi del Po e della Collina Torinese, L'infrastruttura verde del parco del Po torinese, Alinea Editrice, Firenze (2008). 